\title{
Modeling Urea-Water Solution Droplet Evaporation
}

\author{
Douglas T. Ryddner • Mario F. Trujillo
}

Received: 2 September 2014 / Accepted: 1 December 2014 / Published online: 16 January 2015

(c) Springer SIP, AG 2015

\begin{abstract}
A computational solver has been developed for the calculation of urea-water-solution (UWS) droplet vaporization. It is based on the solution of the mass density, chemical species transport, and energy within the droplet, and it is fully coupled to the jump conditions for species and energy transport at the droplet interface, and the phase-equilibrium conditions. Pressure-volumetemperature relationships and fugacities are predicted using the Peng-Robinson equation of state. The numerical code is validated by testing its ability to resolve the dynamics of internal species and temperature fields during phase change, predict phase equilibrium for UWS and hydrocarbon systems, and predict vaporization for $\mathrm{C}_{7} \mathrm{H}_{16}, \mathrm{C}_{7} \mathrm{H}_{16} / \mathrm{C}_{10} \mathrm{H}_{22}$, and UWS droplets. Results show that UWS droplet vaporization can be divided into three different phases consisting of (i) temperature rise at nearly constant composition, (ii) overall urea enrichment at nearly constant temperature, and (iii) simultaneous overall heating and urea enrichment of the entire droplet. The third phase is typically characterized by solidification of the gas-liquid interface, producing a urea shell, a state that can potentially lead to micro-explosions. Higher ambient temperatures are shown to promote urea solidification more readily than lower temperatures to the decreasing role of liquid species diffusion with increasing temperature.
\end{abstract}

Keywords Urea · UWS · Droplet vaporization

D. T. Ryddner · M. F. Trujillo ( $\triangle)$

Department of Mechanical Engineering, University of Wisconsin,

Madison, WI 53706, USA

e-mail: mtrujillo@wisc.edu

\section{Nomenclature}

Normal symbols are used for scalars and boldfaced symbols for vectors

$\begin{array}{ll}\text { Symbol } & \text { Description } \\ A & \text { Peng-Robinson parameter } \\ a_{m} & \text { Peng-Robinson parameter } \\ b_{m} & \text { Peng-Robinson parameter } \\ B_{N u} & \text { Nusselt number parameter } \\ \mathbf{b} & \text { Computational cell displacement } \\ & \text { rate } \\ \mathscr{D}_{a b} & \text { Liquid diffusion between species } \\ D_{i} & \text { Initial diameter } \\ D_{w i r e} & \text { Diameter of wire } \\ f & \text { Fugacity } \\ \Delta H_{f} & \text { Change in enthalpy } \\ L & \text { Length } \\ L_{v a p} & \text { Latent heat of vaporization } \\ M & \text { Molar mass } \\ N_{s p} & \text { Number of species } \\ N_{c e l l s} & \text { Number of cells in domain } \\ P r & \text { Prandtl number } \\ \mathbf{R}_{\mathbf{s}} & \text { Droplet surface regression rate } \\ R & \text { Specific gas constant } \\ S c & \text { Schmidt number } \\ S & \text { Surface area } \\ t & \text { Time } \\ \mathbf{U} & \text { Thermal expansion velocity } \\ v & \text { Specific volume } \\ & \end{array}$




$\begin{array}{ll}\frac{d X(t)}{d t} & \text { Rate of change of position } \\ Z & \text { Compressiblity }=\frac{P v}{R T} \\ a_{j} & \text { Peng-Robinson parameter } \\ B & \text { Peng-Robinson parameter } \\ B_{S h, a} & \text { Sherwood number parameter for } \\ & \text { species a } \\ b_{j} & \text { Peng-Robinson parameter } \\ C_{p} & \text { Specific heat of mixture } \\ D & \text { Diameter } \\ D_{d r o p} & \text { Diameter of experimental droplet } \\ e_{r} & \text { Radial unit vector } \\ H_{\alpha} & \text { Enthalpy } \\ k & \text { Thermal conductivity } \\ L_{f u s} & \text { Latent heat of fusion } \\ L e & \text { Lewis number } \\ m & \text { Peng-Robinson function of } \omega \\ N u & \text { Nusselt number } \\ P & \text { Pressure } \\ Q & \text { Heat of reaction } \\ R_{u n i v} & \text { Gas constant } \\ S h & \text { Sherwood number } \\ T & \text { Temperature } \\ \mathbf{u} & \text { Local fluid velocity that results } \\ V & \text { directly from thermal expansion } \\ X & \text { Volume } \\ Y & \text { Mole fraction } \\ & \text { Mass fraction } \\ & \end{array}$

\section{Greek Symbols}

$\begin{array}{ll}\text { Symbol } & \text { Description } \\ \alpha & \text { Thermal diffusivity } \\ \nu & \text { Kinematic viscosity } \\ \tau & \text { Stress tensor } \\ \tau_{d i f f} & \text { Species diffusion time scale } \\ \phi & \text { Peng-Robinson function of fugacity } \\ \chi & \text { Variable from Abramzon and Sirig- } \\ & \text { nano [1] } \\ \Omega_{j} & \text { Computational cell } \\ \omega & \text { Acentric factor } \\ \kappa & \text { Notation for shorthand governing } \\ \lambda & \text { equations, Table 1 } \\ \rho & \text { Notation from Kucera and Hill [24] } \\ \tau_{C I S} & \text { Density } \\ \tau_{v a p} & \text { Change in slope time } \\ \varphi & \text { Vaporization time scale } \\ \Psi & \text { Notation for shorthand governing } \\ & \text { equations, Table 1 } \\ \partial \Omega_{j} & \text { Notation for shorthand governing } \\ & \text { equations, Table 1 } \\ & \text { Surface of computational cell }\end{array}$

\section{Sub/Superscripts}

$\begin{array}{ll}\text { Symbol } & \text { Description } \\ a & \text { Species a } \\ \text { crit } & \text { Critical property } \\ g & \text { Gas } \\ l & \text { Liquid } \\ \text { mod } & \text { Modified equation } \\ r & \text { Reduced value, i.e., } T / T_{\text {crit }} \\ \text { sat } & \text { Value at saturation pressure } \\ \text { species } & \text { Equation governed by species values } \\ \text { urea } & \text { Urea } \\ \text { air } & \text { Air } \\ \text { eff } & \text { Effective property } \\ \mathrm{H}_{2} \mathrm{O} & \text { Water } \\ \text { mix } & \text { Corresponding to a mixture } \\ \text { partial } & \text { Partial pressure } \\ \text { s } & \text { Value at the gas-liquid interface } \\ \text { sol } & \text { Solid } \\ \text { thermal } & \text { Corresponding to thermal properties } \\ \infty & \text { Properties at infinity }\end{array}$

\section{Introduction}

With ever-tightening pollutant emission regulations [20], a common procedure for removing one of the major components of diesel engine emissions, namely nitric oxides $\left(N O_{x}\right)$, is urea-water-solution (UWS)-based selective catalytic reduction (SCR) [4, 20, 38]. In this method, the UWS spray droplets are exposed to the combustion exhaust stream and are subsequently vaporized producing ammonia $\left(\mathrm{NH}_{3}\right)$ and isocyanic acid (HNCO) through a thermolysis reaction [32]. It is the $\mathrm{NH}_{3}$ which serves as a reducing agent to transform $\mathrm{NO}_{x}$ into $\mathrm{N}_{2}$ on the catalyst. Schaber et al. [32] also mentions other reactions which occur within the desired temperature regime as well, including creation of undesirable chemical compounds; biuret, cyanuric acid, ammelide, and ammeline complexes. One of the strongest benefits of this procedure is the safe storage of $\mathrm{NH}_{3}$ within the UWS. Due to their potential, UWS and urea have been the subject of extensive research $[5,14,15,27,32,39]$ with regards to

Table 1 Variables for the generalized conservation law

\begin{tabular}{llll}
\hline Equations & $\kappa$ & $\varphi$ & $\Psi$ \\
\hline Mass & 1 & 1 & 0 \\
Species & 1 & $Y_{a}$ & $\rho D_{a b} \nabla Y_{a}$ \\
Energy & $c_{p}$ & $T$ & $k \nabla T$ \\
\hline
\end{tabular}


physical characteristics, chemical decomposition pathways, evaporation time, and gas phase properties.

In conjunction with the aforementioned experimental studies, a number of computational efforts have also been presented $[2,3,7,11,21,25,26,35]$, particularly in the last few years. One common topic of investigation is the choice between depletion models, which includes a vaporization treatment for both urea and water, and a vaporization treatment for water followed by direct thermolysis of urea. It has been shown, in the work of Abu-Ramadan et al. [3], that the vaporization approach generates more accurate predictions. Furthermore, the treatment of direct thermolysis from the aqueous state into the gas phase has been criticized by Lundstrom et al. [25] as predicting droplet temperatures that were excessively high. More evidence favoring the vaporization route has also been presented by the experiments of Bernhard et al. [5], where they advocate the consideration of gaseous urea in computational models.

A common view adopted in many of the modeling efforts describes the gasification of urea from UWS droplets as occurring in two phases, as documented by Kim et al. [21]. In the first phase, water is completely vaporized from the droplet in a process that ignores the presence of urea. Once the water has been vaporized, the second phase considers the gasification of urea, which takes place as low as $406 \mathrm{~K}$ according to data from Bernhard et al. [5] and Emel'yanenko et al. [14]. This physical picture is adopted in the work of Birkhold et al. [7], Ström et al. [35], Munnannur and Liu [26], Ebrahimian et al. [11], and Lundstrom et al. [25]. In a recent publication, AbuRamadan et al. [3] raises questions regarding the validity of this interpretation; the authors provide a compelling physical explanation of the gradual enrichment of urea at the gas-liquid interface as water vaporizes preferentially, due to its lower boiling point. Eventually, this leads to precipitation of urea at the gas-liquid interface, which essentially traps a fraction of the water inside the droplet. This results in a subsequent increase in the surface temperature of the droplet in combination with vaporization of urea. Hence, the water is not fully vaporized prior to the initiation of urea gasification.

Associated with the notion of a complete water vaporization phase followed by a gasification of urea, Birkhold et al. [7] presented some insightful results contrasting the vaporization behavior between the diffusion-limited (DL) model and the rapid-mixing (RM) model. These approaches had been presented earlier, but within the context of multicomponent droplet vaporization aimed at hydrocarbon mixtures, as elaborated by Sirignano [33]. Birkhold et al. [7] conclude that both of these models give predictions that are very similar to each other, especially with regards to $D^{2}(t)$ histories, but since the RM model is significantly computationally cheaper, their results provide an attractive reason for adopting it. Unfortunately, the RM model is essentially an infinite diffusivity assumption, and as such is incapable of predicting the preferential concentration of urea at the gas-liquid interface. As an important consequence, it cannot predict the entrapment of water caused by the precipitation of urea at the droplet surface. Furthermore, the discrepancy between models is only negligible while water is still actively vaporizing from the interface. As soon as the urea shell is formed, the discrepancy becomes significant.

In the present effort, we adopt a multicomponent treatment of the UWS droplet, where both urea and water are resolved within the droplet. This treatment is then coupled to the jump conditions for species and energy at the gas-liquid interface, and to a set of vapor-liquid equilibrium relations for each of the chemical species. Results from the calculations clearly show the evolution of the mass fraction profiles at each radial location, leading to a complete enrichment of urea at the gas-liquid interface, which is also seen by Abu-Ramadan et al. [3]. Predictions from our calculations agree well with single species droplet vaporization studies of $\mathrm{C}_{7} \mathrm{H}_{16}$ from Nomura et al. [28], the multicomponent fuel droplet results from Daif et al. [10], and experimental data of UWS droplet vaporization from Wang et al. [39]. This work then explores the physical processes that can lead to micro-explosions inside the droplet.

Though the nature of this work bears similarities with the previous work on UWS vaporization, including work of Abu-Ramadan et al. [3], Ebrahimian et al. [11], and Lundstrom et al. [25], notable improvements and additions are included. Explicitly, these are as follows: (i) the evolution of the thermodynamic state of the droplet is presented as a spatially dependent quantity within the urea-water phase diagram, (ii) the numerical algorithm solves the entire interfacial system simultaneously (jump conditions and phase equilibrium) fully coupled to the corresponding droplet internal numerical solution, (iii) the multicomponent system is described by the Peng-Robinson equation of state, providing solutions for the phase equilibrium conditions, as well as predicting the pressure-volumetemperature (PVT) relations, (iv) the surface solidification of urea is tracked for the droplet lifetime, and (v) the effect of internal mixing resulting from aerodynamic drag is studied.

In the following subsections, the relevant governing equations are presented along with the equation of state, and the thermophysical properties. The interfacial constraints, which include the jump conditions and phase equilibrium relations are described. This is followed by 
a description of the numerical algorithm, which is subsequently verified against analytical results. Validation of the code is presented for UWS phase-equilibrium calculations, $\mathrm{C}_{7} \mathrm{H}_{16}$ droplet vaporization, and $\mathrm{C}_{7} \mathrm{H}_{16} / \mathrm{C}_{10} \mathrm{H}_{22}$ bicomponent droplet vaporization. Finally, the UWS droplet vaporization computations are contrasted with experimental findings from Wang et al. [39]. This leads into an analysis of the evolution of thermodynamic states within the droplet. The main findings are then summarized in the conclusion.

\section{Governing Equations}

The following conservation equations for mass, species, and energy for a multicomponent liquid mixture [6] apply within the droplet:

$$
\begin{aligned}
\frac{\partial \rho}{\partial t}+\nabla \cdot(\rho \mathbf{u})= & 0, \\
\frac{\partial\left(\rho Y_{a}\right)}{\partial t}+\nabla \cdot\left(\rho Y_{a} \mathbf{u}\right)= & \nabla \cdot\left(\rho \mathscr{D}_{a b} \nabla Y_{a}\right), \\
\rho C_{p} \frac{D T}{D t}= & \left.\frac{\partial(\ln v)}{\partial(\ln T)}\right|_{P, Y_{a}} \frac{D P}{D t}+\tau: \nabla \mathbf{u} \\
& +\nabla \cdot(k \nabla T) \\
& +\sum_{a=1}^{N_{s p}}\left(\rho \mathscr{D}_{a b} \nabla Y_{a}\right) \cdot \nabla H_{\alpha} .
\end{aligned}
$$

Additionally, the following constraints are enforced in either the liquid or gas phase

$$
\sum_{a=1}^{N_{s p}} X_{a}^{g, l}=1 \quad \text { and } \quad \sum_{a=1}^{N_{s p}} Y_{a}^{g, l}=1,
$$

where the relation between mass and molar fractions is

$$
Y_{a}=\frac{M_{a} X_{a}}{\sum_{i=1}^{N_{s p}} M_{i} X_{i}} .
$$

The contribution of the viscous dissipation term can be considered to be negligible in the energy equation. The Lagrangian derivative for pressure is negligible as well, as the droplet considered experiences no external forces. Additionally, earlier computations for the type of systems considered here, i.e., UWS, showed that the enthalpy diffusion term can also be neglected. With these simplifications and rewriting, the left-hand side of the energy equation in conservative form yields

$C_{p}\left[\frac{\partial(\rho T)}{\partial t}+\nabla \cdot(\rho \mathbf{u} T)\right]=\nabla \cdot\left(k^{l} \nabla T\right)$.

A spherically symmetric system is employed in our work, where the effects of internal liquid mixing are incorporated with the effective species and heat diffusion models of $[1,33]$ :

$$
\begin{aligned}
\mathscr{D}_{\text {ab,eff }} & =\left(\chi_{\text {species }}\right) \mathscr{D}_{a b}, \\
k_{\text {eff }}^{l} & =\left(\chi_{\text {thermal }}\right) k^{l}, \quad \text { where } \\
\chi_{\text {species }} & =1.86+0.86 \tanh \left(2.225 \log _{10}\left(\frac{R e^{l} S c}{30}\right)\right), \text { and }(5 \mathrm{c}) \\
\chi_{\text {thermal }} & =1.86+0.86 \tanh \left(2.225 \log _{10}\left(\frac{R e^{l} P r}{30}\right)\right) .
\end{aligned}
$$

The non-dimensional quantities are given by,

$R e^{l}=\frac{2 R_{s} u}{v}, \quad S c=\frac{v}{\mathscr{D}_{a b}}$, and $\quad \operatorname{Pr}=\frac{\nu}{\alpha}$.

This transforms the previous form of the governing equations to the following set

$$
\begin{aligned}
\frac{\partial \rho}{\partial t}+\nabla \cdot(\rho \mathbf{u}) & =0, \\
\frac{\partial\left(\rho Y_{a}\right)}{\partial t}+\nabla \cdot\left(\rho Y_{a} \mathbf{u}\right) & =\nabla \cdot\left(\rho \mathscr{D}_{a b, e f f} \nabla Y_{a}\right), \\
C_{p}\left[\frac{\partial(\rho T)}{\partial t}+\nabla \cdot(\rho T \mathbf{u})\right] & =\nabla \cdot\left(k_{e f f}^{l} \nabla T\right) .
\end{aligned}
$$

This is the group of equations that is subsequently discretized and solved in the present work. The advection term is kept in the solution procedure, since it accounts for thermal expansion. This effect is noticeable in the initial thermal transient when the droplet is undergoing significant heating.

\subsection{Peng-Robinson Equation of State}

The Peng-Robinson equation of state (PR-EOS) is employed [29] in the present work to predict pressurevolume-temperature (PVT) relationships for both gas and liquid phases, and to predict fugacities needed for phase equilibrium calculations. The PR-EOS is chosen due to its favorable balance between computational simplicity and accuracy [19, 41]. Its behavior for our particular problem is tested in Section 4.2. The cubic form of the Peng-Robinson EOS is given in terms of the compressibility factor $(Z=$ $P v /(R T))$ as $[22,37]$

$$
Z^{3}-(1-B) Z^{2}+\left(A-3 B^{2}-2 B\right) Z-\left(A B-B^{2}-B^{3}\right)=0,
$$

where A, B, and all other relevant PR-EOS variables are defined in Appendix A. Densities are directly calculated from $Z$. Phase equilibrium is calculated by equating liquid and gas fugacities, where the fugacity for either gas or liquid is given respectively as

$f_{a}^{g}=\phi_{a}^{g} P X_{a, s}^{g}, \quad$ or $\quad f_{a}^{l}=\phi_{a}^{l} P X_{a, s}^{l}$, 
for some chemical species $a$. The associated fugacity coefficient, for instance the liquid one, is defined as

$$
\begin{aligned}
\ln \left(\phi_{a}^{l}\right)= & \frac{b_{a}}{b_{m}^{l}}\left(Z^{l}-1\right)-\log \left(Z^{l}-B^{l}\right)-\left(\frac{A^{l}}{2 \sqrt{2} B^{l}}\right) \\
& \left(\sum_{j=1}^{N_{s p}} \sum_{k=1}^{N_{s p}} 2 X_{m, j}^{l}\left(1-k_{j k}\right)\left(\frac{\sqrt{a_{j} a_{k}}}{a_{m,}^{l}}-\frac{b_{j}}{b_{m,}^{l}}\right)\right) \\
& \log \left(\frac{Z^{l}+(\sqrt{2}+1) B^{l}}{Z^{l}-(\sqrt{2}-1) B^{l}}\right) .
\end{aligned}
$$

The same expression holds for the gas phase fugacity coefficient with the gas quantities substituted in place of the liquid ones.

\subsection{Interfacial Constraints}

The equations solved at the droplet surface form a set of six coupled, nonlinear equations, namely three for the phase equilibrium conditions for urea, water, and air, one for the energy-jump condition, and two for the species jump condition for urea and water. The equations are:

$$
\begin{aligned}
\phi_{\text {urea }}^{g} X_{\text {urea }, s}^{g} P & =\phi_{\text {urea }}^{l} X_{\text {urea }, s}^{l} P \\
\phi_{H_{2} O}^{g} X_{H_{2} O, s}^{g} P & =\phi_{H_{2} O}^{l} X_{H_{2} O, s}^{l} P, \\
\phi_{\text {air }}^{g}\left(1-X_{H_{2} O, s}^{g}-X_{\text {urea }, s}^{g}\right) P & =\phi_{\text {air }}^{l}\left(1-X_{H_{2} O, s}^{l}-X_{\text {urea }, s}^{l}\right) P, \\
\rho^{l}\left(\dot{R}_{s}-U_{s}\right) L_{\text {vap }, \text { mix }} & =\left[k_{\text {mix }}^{l} \frac{d T}{d r}\right]_{r=R_{s}}^{l}-\frac{N u}{2 R_{s}} k_{\text {mix }}^{g}\left(T_{s}-T_{\infty}\right), \\
\rho_{s}^{l}\left(Y_{\text {urea }, s}^{g}-Y_{\text {urea }, s}^{l}\right)\left(\dot{R}_{s}-U_{s}\right) & =-\left(\rho_{s}^{g} \mathscr{D}_{\text {urea air air }}^{g} \frac{S h}{2 R_{S}}\left(Y_{\text {urea }, \infty}^{g}-Y_{\text {urea }, s}^{g}\right)-\left.\rho_{s}^{l} \mathscr{D}_{\text {urea }, H_{2} 0}^{l} \frac{d Y_{s, \text { urea }}}{d r}\right|_{r=R_{s}} ^{l}\right), \\
\rho_{s}^{l}\left(Y_{H_{2} O, s}^{g}-Y_{H_{2} O, s}^{l}\right)\left(\dot{R}_{s}-U_{s}\right) & =-\left(\rho_{s}^{g} \mathscr{D}_{H_{2} O, \text { air }}^{g} \frac{S h}{2 R_{S}}\left(Y_{H_{2} O, \infty}^{g}-Y_{H_{2} O, s}^{g}\right)-\left.\rho_{s}^{l} \mathscr{D}_{\text {urea }, H_{2} O}^{l} \frac{d Y_{s, H_{2} O}}{d r}\right|_{r=R_{s}} ^{l}\right),
\end{aligned}
$$

where pressure cancels in the phase equilibrium relations, and the gradients from the gas-side of the interface have been expressed using

$$
\begin{gathered}
\left.\frac{\partial T}{\partial r}\right]_{r=R_{s}}^{g}=\frac{N u}{2 R_{s}} k_{\text {mix }}^{g}\left(T_{s}-T_{\infty}\right), \\
\left.\rho_{s}^{g} \mathscr{D}_{a b}^{g} \frac{\partial Y_{a}}{\partial r}\right|_{r=R_{s}} ^{g}=\rho_{s}^{g} \mathscr{D}_{a b}^{g} \frac{S h}{2 R_{S}}\left(Y_{\infty, a}^{g}-Y_{s, a}^{g}\right) .
\end{gathered}
$$

The Nusselt and Sherwood numbers are given by,

$N u=\frac{2 \log \left(1+B_{N u}\right)}{B_{N u}}, \quad$ and $\quad S h_{a}=\frac{2 \log \left(1+B_{S h, a}\right)}{B_{S h, a}}$,

where [33]

$B_{N u}=\frac{C_{p}\left(T_{\infty}-T_{S}\right)}{L_{e f f}}, \quad$ and $\quad B_{S h, a}=\frac{Y_{a}^{g}}{1-Y_{a}^{g}}$.

The property values in the above non-dimensional numbers, which correspond to the gas phase, are calculated at the temperature and species mass fraction corresponding to the arithmetic mean rule,

$T^{g}=T_{s}+A_{r}\left(T_{\infty}-T_{s}\right), \quad Y^{g}=Y_{s}+A_{r}\left(Y_{\infty}-Y_{s}\right)$, where $A_{r}$ is either $1 / 2$ or $1 / 3$ [9]. In the calculations presented here, we employ $A_{r}=1 / 3$.

The resulting system (11a-f) introduces ten unknowns, namely,

$\left[T_{s}, \dot{R}_{s}, X_{s, H_{2} O}^{l}, X_{s, H_{2} O}^{g}, X_{s, \text { urea }}^{l}, X_{s, \text { urea }}^{g}, X_{s, \text { air }}^{l}, X_{s, \text { air }}^{g}, \rho_{s}^{l}, \rho_{s}^{g}\right]$.

The PR-EOS, (8), provides a solution for the liquid and gas phase density, reducing the unknowns to eight. The conservation of species from Eq. 2 provides two additional constraints, $X_{a i r}^{l, g}=1-X_{H_{2} O}^{l, g}-X_{\text {urea }}^{l, g}$, for gas and liquid, reducing the unknowns to

$\left[T_{s}, X_{s, \mathrm{H}_{2} \mathrm{O}}^{l}, X_{s, \mathrm{H}_{2} \mathrm{O}}^{g}, X_{s, \text { urea }}^{l}, X_{s, \text { urea }}^{g}, \dot{R}_{s}\right]$.

At the center of the droplet $(r=0)$, both radial derivatives of temperature and species mass fraction are set to zero.

\subsection{Modifications to the Latent Heat of Vaporization}

Due to the short time lapse between urea vaporization [5] and decomposition [32] into $\mathrm{NH}_{3}$ and $\mathrm{HNCO}$, and the fact that the decomposition represents an endothermic process, we model both of these process as taking place 
simultaneously. This implies that in the gasification treatment, a modified latent heat is employed, which is given by

$L_{\text {vap, } \text { urea,mod }}=Q_{\text {decomposition }}+L_{\text {vap, } \text { urea }}$.

The enthalpy of decomposition, $Q_{\text {decomposition }}$, corresponds to the following reaction

$\left[\mathrm{CH}_{4} \mathrm{~N}_{2} \mathrm{O}\right]^{g} \rightarrow\left[\mathrm{NH}_{3}+\mathrm{HNCO}^{g}\right.$ (endothermic),

and is calculated from

$$
\begin{aligned}
Q_{\text {decomposition }}= & {\left[\Delta H_{f, \text { urea }}+C_{p, \text { urea }} \Delta T\right] } \\
& -\left[\Delta H_{f, N H 3}+C_{p, N H 3} \Delta T\right. \\
& \left.+\Delta H_{f, H N C O}+C_{p, H N C O} \Delta T\right] .
\end{aligned}
$$

This relation represents the difference between heat of the products and reactants. $\Delta H_{f, a}$ pertains to the heat of formation for chemical species $a$, and $\Delta T=T_{s}-T_{o}$, where $T_{o}=297 \mathrm{~K}$ is a reference temperature. A method similar to this is implemented by both Birkhold et al. [7] and Ström et al. [35]. We also note that applying the endothermic process of decomposition to the latent heat of vaporization simulates the process of the decomposition reaction taking energy out of the system in the boundary layer of the droplet.

\subsection{Modeling the Solidification Front of Urea}

UWS droplet vaporization experiments by Kontin et al. [23], Musa et al. [27], and Wang et al. [39] provide evidence for the solidifying urea on the surface of the droplet. This study takes the solidification of urea into account while numerically calculating the vaporization of the droplet. The process is modeled by changing the physical properties of a liquid mixture to one corresponding to a liquid-solid mixture, consistent with the urea-solidification region (see Fig. 9 and surrounding text, as well as Appendix B). The corresponding computational cell-averaged physical properties, which are the ones needed in the solution of governing equations, are obtained from the lever rule [13].

\section{Numerical Solution}

The conservation Eqs. 7a, 7b, and 7c can be written in the following general form

$\frac{\partial(\rho \varphi)}{\partial t}+\nabla \cdot(\rho \varphi \mathbf{u})=\frac{1}{\kappa} \nabla \cdot \Psi$,

where the dependent variables are given in Table 1. Integration of this general conservation law over an arbitrary computational cell, $\left(\Omega_{j}\right)$ gives

$\int_{\Omega_{j}} \frac{\partial(\rho \varphi)}{\partial t} d V+\int_{\partial \Omega_{j}} \rho \varphi \mathbf{u} \cdot \mathbf{n} d S=\int_{\Omega_{j}} \frac{1}{\kappa} \nabla \cdot \Psi d V$.
The numerical solution is based on a combination of finite volume discretization combined with ArbitraryLagrangian-Eulerian procedure for treating the unsteady and advection terms [36]. Following this procedure, we define an arbitrary velocity field (spherically symmetric) as $\mathbf{b}(r, t)=\left(\frac{r}{R_{S}}\right) \dot{R}_{s} \mathbf{e}_{r}$.

The Lagrangian derivative following $\mathbf{b}$ is then given by

$$
\left.\frac{D}{D t}\right|_{\mathbf{b}}\left(\int_{\Omega_{j}} \rho \varphi d V\right)=\int_{\Omega_{j}} \frac{\partial(\rho \varphi)}{\partial t} d V+\int_{\partial \Omega_{j}} \rho \varphi \mathbf{b} \cdot \mathbf{n} d S .
$$

With the help of this relation, we can rewrite (22) as

$$
\left.\frac{D}{D t}\right|_{\mathbf{b}}\left(\int_{\Omega_{j}} \rho \varphi d V\right)+\int_{\partial \Omega_{j}} \rho \varphi(\mathbf{u}-\mathbf{b}) \cdot \mathbf{n} d S=\int_{\Omega_{j}} \frac{1}{\kappa} \nabla \cdot \Psi d V,
$$

or in discretized form as

$$
\begin{aligned}
\frac{(\rho \varphi V)_{j}^{n+1}-(\rho \varphi V)_{j}^{n}}{\Delta t}= & \left.\{\rho \varphi(b-u) S\}\right|_{j-1 / 2, n} ^{j+1 / 2, n} \\
& +\left.\frac{1}{\bar{\kappa}}\{\Psi S\}\right|_{j-1 / 2, n} ^{j+1 / 2, n},
\end{aligned}
$$

where $V_{j}^{n}$ is defined as

$V_{j}^{n}=\left[\frac{r_{j+1 / 2}^{3}-r_{j-1 / 2}^{3}}{3}\right]^{n}$.

Here, an explicit Euler time stepping scheme has been employed with time level being indicated by $n$. The computational cell index is $j \in\left(0, N_{\text {cells }}\right)$ and corresponds to the cell center. Indices $(j+1 / 2)$ or $(j-1 / 2)$ correspond to cell faces (Fig. 1). Additionally, an arithmetic mean value for $(1 / \kappa)$ is performed to reduce the diffusion flux term to the presented discretized form. From Eq. 26, the explicit expressions for density, mass fraction, and temperature become:

$$
\begin{aligned}
\rho_{j}^{n+1}= & \frac{V_{j}^{n}}{V_{j+1}^{n}} \rho_{j}^{n}+\left.\frac{\Delta t}{V_{j+1}^{n}}\{\rho(b-u) S\}\right|_{j-1 / 2, n} ^{j+1 / 2, n}, \\
Y_{j, a}^{n+1}= & \frac{\left(\rho Y_{a} V\right)_{j}^{n}}{(\rho V)_{j}^{n+1}}+\frac{\Delta t}{(\rho V)_{j}^{n+1}}\left(\left.\left\{\rho Y_{a}(b-u) S\right\}\right|_{j-1 / 2, n} ^{j+1 / 2, n}\right. \\
& \left.+\left.\frac{1}{\bar{\kappa}}\left\{\left(\rho D_{a b} \nabla Y_{a}\right) S\right\}\right|_{j-1 / 2, n} ^{j+1 / 2, n}\right) \\
T_{j}^{n+1}= & \frac{(\rho T V)_{j}^{n}}{(\rho V)_{j}^{n+1}}+\frac{\Delta t}{(\rho V)_{j}^{n+1}}\left(\left.\{\rho T(b-u) S\}\right|_{j-1 / 2, n} ^{j+1 / 2, n}\right. \\
& \left.+\left.\frac{1}{\bar{\kappa}}\{(k \nabla T) S\}\right|_{j-1 / 2, n} ^{j+1 / 2, n}\right)
\end{aligned}
$$


Fig. 1 Graphical representation of the arrangement of variables within the spherically symmetric grid. Temperature, species mass fraction, and density are cell-centered, and grid node displacement and flow velocity are defined at the faces

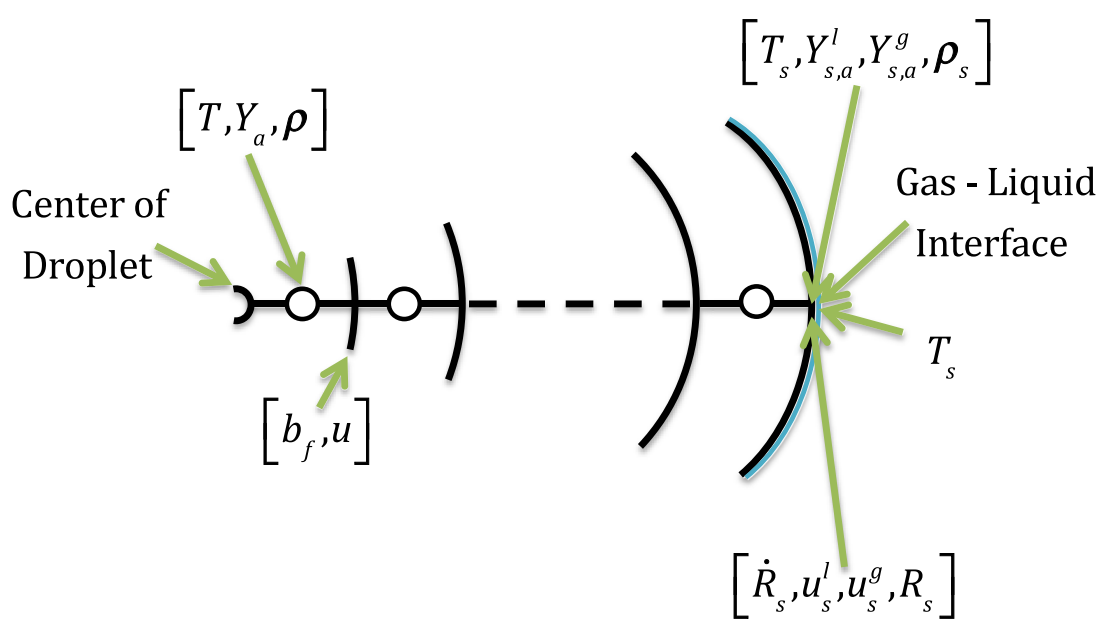

The resulting algorithm employed in the calculations can be summarized as follows:

I. Velocity field is initialized uniformly to zero. The urea and water mass fraction are also uniformly set to their eutectic values, namely $Y_{\text {urea }}=0.325$ and $Y_{H_{2} \mathrm{O}}=0.675$. Within the droplet, the temperature has a constant value equal to $300 \mathrm{~K}$ and the pressure is set to $1 \mathrm{~atm}$. The PR-EOS is employed to calculate the density field. All thermophysical quantities are calculated.

II. The interfacial system described by Eqs. 11a-f is computed resulting in new values for surface temperature and chemical compositions on both the liquid and gas side of the interface, as well as a new surface regression rate, $\dot{R}_{s}$.

III. Based on the calculated $\dot{R}_{S}$, the flow field $b(r, t)(23)$ is updated to calculate $V_{j}^{n+1}(27)$.

IV. Mass density iteration

a. Density field is calculated based on the PR-EOS, namely $\rho_{j}^{n+1}=P R-\operatorname{EOS}\left(Y_{j, a}^{n+1}, T_{j, a}^{n+1}, P\right) .{ }^{1}$ Using the mass density (28a) and this computed value for $\rho_{j}^{n+1}$, the flow field $\mathbf{u}_{j \pm 1 / 2}$ is calculated, where $\mathbf{u}_{1 / 2}=0$.

b. Compute $Y_{j, a}^{n+1}$ from Eq. 28 b.

c. Compute $T_{j}^{n+1}$ from Eq. 28c.

d. Check whether the maximum fractional change in density between successive iterations and corresponding to all computational cells exceeds $10^{-10}$. If it does return to (a). Otherwise, exit mass density iteration loop.

V. If the calculation has completed the required number of time iterations, exit. Otherwise, return to II above.

$\overline{{ }^{1} \text { In the first iteration } \rho_{j}^{n+1}=P R-E O S\left(Y_{j, a}^{n}, T_{j, a}^{n}, P\right)}$
The computations take approximately $0.09 \mathrm{~s}$ per time step, and the maximum number of time steps used is around 0.5 million for the entire droplet vaporization (this algorithm is not presently optimized for speed). Due to the explicitness of the scheme, time step size is modified to remain stable with respect to diffusion discretization and the CFL condition.

\section{Results}

Prior to the calculations of UWS droplet gasification, the various aspects of the solver are systematically tested and presented in the following sections. First, the solution pertaining to the internal fields is tested against analytical solutions in Section 4.1. This is followed by an assessment of phase equilibrium predictions in Section 4.2. Validation for droplet vaporization predictions are presented for single liquid species $\left(\mathrm{C}_{7} \mathrm{H}_{16}\right)$ and binary liquid species systems in Sections 4.3 and 4.4, respectively.

\subsection{Phase Change and Heat Transfer Validation}

To evaluate the internal solution methodology, a comparison is made against two heat transfer problems with phase change [24]. The first problem consists of a planar 1D system in a domain $-\infty<z \leq L$, where a solid medium exists at some uniform initial temperature $T_{i}$. Immediately after $t=0$, the temperature at $z=L$, i.e., $T(L, t>0)$ is set equal to $T_{a}$, where $T_{a}$ is greater than the fusion temperature $\left(T_{f u s}\right)$. This produces a liquid region, where the liquidsolid interface is tracked by $X(t)$. For the second problem, a spherically symmetric domain is considered, $0 \leq z \leq L$, where again a solid medium exists at a uniform temperature $T_{i}$. At $t^{+}=0, T(L, t>0)=T_{a}$ and the melt layer 
Fig. 2 Comparison of temperature predictions against analytical solutions from Kucera and Hill [24] corresponding to the a planar case $\left(t=2.2 \times 10^{-5}\right.$ s) and $\mathbf{b}$ spherical case $\left(t=1.45 \times 10^{-5} \mathrm{~s}\right)$

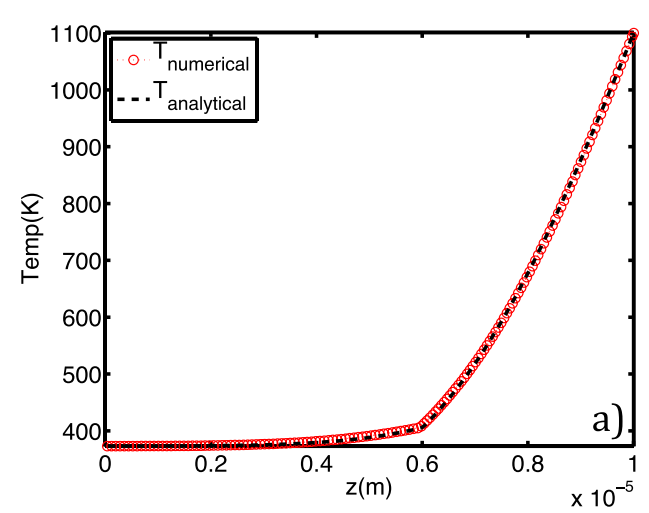

propagates inwardly. The governing equations for both problems are given by

$$
\begin{aligned}
& \frac{\partial T}{\partial t}=\frac{k^{l}}{\rho C}\left(\frac{\partial^{2} T}{\partial z^{2}}+\frac{\lambda}{z} \frac{\partial T}{\partial z}\right) \quad \text { for } \quad X(t) \leq z<L \quad \text { (liquid) (29a) } \\
& \frac{\partial T}{\partial t}=\frac{k^{s o l}}{\rho C}\left(\frac{\partial^{2} T}{\partial z^{2}}+\frac{\lambda}{z} \frac{\partial T}{\partial z}\right) \quad \text { for } \quad z \leq X(t) \text { (solid). }
\end{aligned}
$$

For the Cartesian and Spherical systems, $\lambda=0$ or $\lambda=2$, respectively. These partial differential equations are coupled to the energy jump condition,

$$
\left(\left.k^{l} \frac{\partial T}{\partial z}\right|_{(X(t), t)}-\left.k^{s o l} \frac{\partial T}{\partial z}\right|_{(X(t), t)}\right)=\rho L_{f u s} \frac{d X(t)}{d t} .
$$

Both density and specific heats are the same in both liquid and solid regions. Conductivity is the only property that varies.

Analytical solutions for the planar and spherical systems are described in detail by Kucera and Hill [24], and due to their elaborate form are not reproduced here. The comparisons between the predictions of our numerical system and these analytical solutions are shown in Fig. 2 for both Cartesian and Spherical systems. In our numerical predictions, we extend the numerical discretization of the temperature field on both sides of the interface using the same scheme presented in Section 3. In these calculations, the following quantities are used: $T_{a}=1100 \mathrm{~K}, T_{f u s}=406 \mathrm{~K}, T_{i}=373 \mathrm{~K}$, $\rho=1320 \mathrm{~kg} / \mathrm{m}^{3}$, and $C=1450 \mathrm{~J} / \mathrm{kg}-\mathrm{K}$. For the planar case $k^{s o l}=0.28 \mathrm{~W} / \mathrm{m}-\mathrm{K}, k^{l}=0.33 \mathrm{~W} / \mathrm{m}-\mathrm{K}$, and $L_{f u s}=$ $2.31 \times 10^{5} \mathrm{~J} / \mathrm{kg}$ are used; while for the spherical case $k^{\text {sol }}=$ $k^{l}=1.0 \mathrm{~W} / \mathrm{m}-\mathrm{K}$, and $L_{f u s}=1 \times 10^{3} \mathrm{~J} / \mathrm{kg}$. As shown in Fig. 2, good agreement is achieved between the numerical and analytical solutions. Furthermore, the discontinuity in the gradient of temperature at the interface is well captured by the present numerical method.

\subsection{Phase Equilibrium Validation}

To evaluate phase equilibrium calculations against ureaair experiments, ambient total pressure is held fixed and the temperature is systematically varied. The associated equations being solved are the phase equilibrium relations (11a), where the quantity being evaluated is the fugacity, i.e., $f_{\text {urea }}^{g, l}=\phi_{\text {urea }}^{g, l} X_{\text {urea }, s}^{g, l} P$. Comparisons to experiments from Bernhard et al. [5] and Emel'yanenko et al. [14] are shown in Fig. 3. Additionally, predictions from the Clausius Clapeyron EOS are also compared, where the relevant equation is

$$
\frac{P_{\text {partial }}}{P}=\exp \left[-\frac{L_{v a p}}{R}\left(\frac{1}{T_{s}}-\frac{1}{T_{\text {sat }}}\right)\right] .
$$

The Clausius-Clapeyron EOS is coupled with Raoult's law to relate species mole fraction to the partial pressure.
Fig. 3 Phase-equilibrium comparisons to experiments a regular scale, b log-y scale. For Bernhard et al. [5], experiments are carried out in gaseous environment of $10 \% \mathrm{O}_{2}$ in $\mathrm{N}_{2}$, and $10 \% \mathrm{O}_{2} 5 \% \mathrm{H}_{2} \mathrm{O}$ in $\mathrm{N}_{2}$. For Emel'yanenko et al. [14], experiments are carried out in gaseous environment of $100 \%$ $\mathrm{N}_{2}$
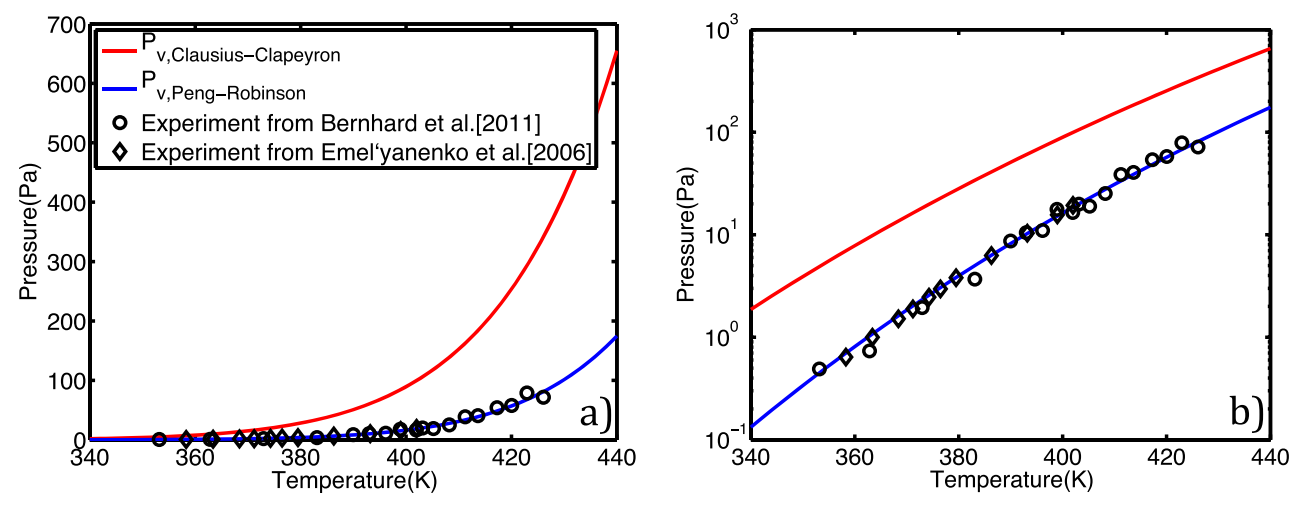


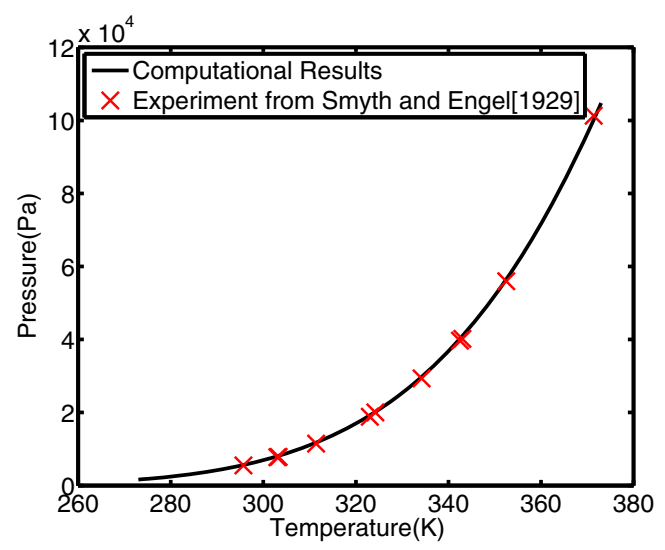

Fig. 4 Phase-equilibrium comparisons for nHeptane-air system

The results are compared against data and shown in Fig. 3a, $\mathrm{b}$, respectively, revealing that the PR-EOS is able to predict fairly well phase equilibrium conditions in comparison to the more ideal treatment given by Clausius-Clapeyron equation. A further comparison is made against the partial pressure data of Smyth and Engel [34] for $\mathrm{C}_{7} \mathrm{H}_{16}$, shown in Fig. 4, with excellent agreement with PR-EOS predictions. Additionally, a comparison against steam table partial pressure data has also been made resulting in good agreement; however, these results are excluded for brevity.

\section{3 $\mathrm{C}_{7} \mathrm{H}_{16}$ Droplet Vaporization in Air}

Having validated the phase equilibrium predictions and the internal solution of the energy equation, the entire system coupled to the interfacial constraints is presently solved to predict the vaporization of a $\mathrm{C}_{7} \mathrm{H}_{16}$ droplet. The results presented in terms of normalized $\mathrm{D}^{2}$ time histories are shown in Fig. 5. They are compared to two sets of data from the work of Nomura et al. [28] in a nitrogen environment at $P=1$ bar. The initial droplet sizes [28] are 0.6 to $0.8 \mathrm{~mm}$ in diameter, and the fiber diameter is $.15 \mathrm{~mm}$ in diameter. Ambient atmospheric pressure characterizes the environment in both

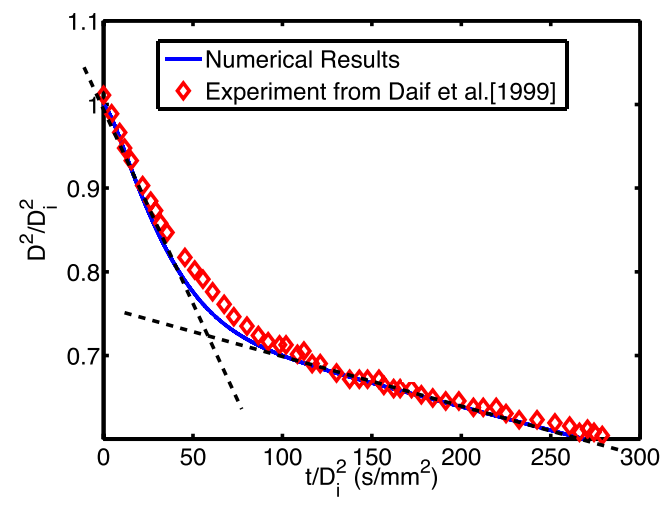

Fig. 6 Comparison between computed and measured $\mathrm{D}^{2}$ time histories corresponding to a $\mathrm{C}_{7} \mathrm{H}_{16} / \mathrm{C}_{10} \mathrm{H}_{22}$ droplet vaporizing in quiescent air at $T=297 \mathrm{~K}$

experimental cases with low and high temperature cases corresponding to $(471 \mathrm{~K})$ and $(741 \mathrm{~K})$, respectively.

While the numerical predictions do not fall exactly on top of the experimental data, the agreement is sufficiently good to inspire confidence in the model. It should be noted from the work of Chauveau et al. [8] that variations in the thickness of the wires used to suspend droplets in these types of experiments can induce a noticeable level of uncertainty in the measurements. It is in light of these experimental uncertainties that the present comparisons are evaluated. Nevertheless, an additional case consisting of a bi-component hydrocarbon mixture is used to further test the code.

\subsection{Bicomponent $\left(\mathrm{C}_{7} \mathrm{H}_{16} / \mathrm{C}_{10} \mathrm{H}_{22}\right)$ Droplet Vaporization}

$\mathrm{D}^{2}$ time history measurements reported by Daif et al. [10] are compared against our numerical predictions and displayed in Fig. 6. The experiment consists of a twocomponent droplet of $50 \% \mathrm{C}_{7} \mathrm{H}_{16}$ and $50 \%$ decane (by volume) vaporizing in quiescent air at $T=297 \mathrm{~K}$, and is performed under normal gravity conditions. Excellent agreement is observed, where a clear transition is displayed
Fig. 5 Comparison to $\mathrm{C}_{7} \mathrm{H}_{16}$ vaporization results at $P=1 \mathrm{bar}$ and at $\mathbf{a} T_{\infty}=471 \mathrm{~K}$ and $T_{\infty}=741 \mathrm{~K}$
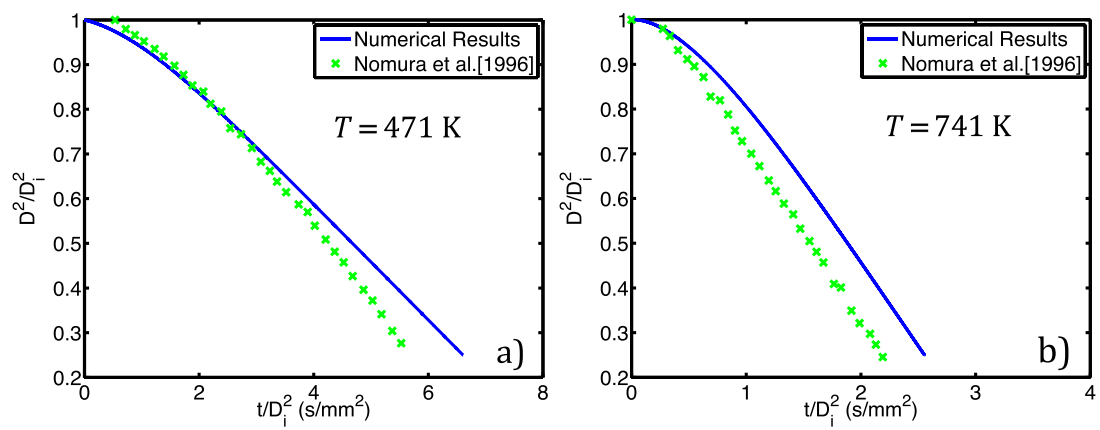

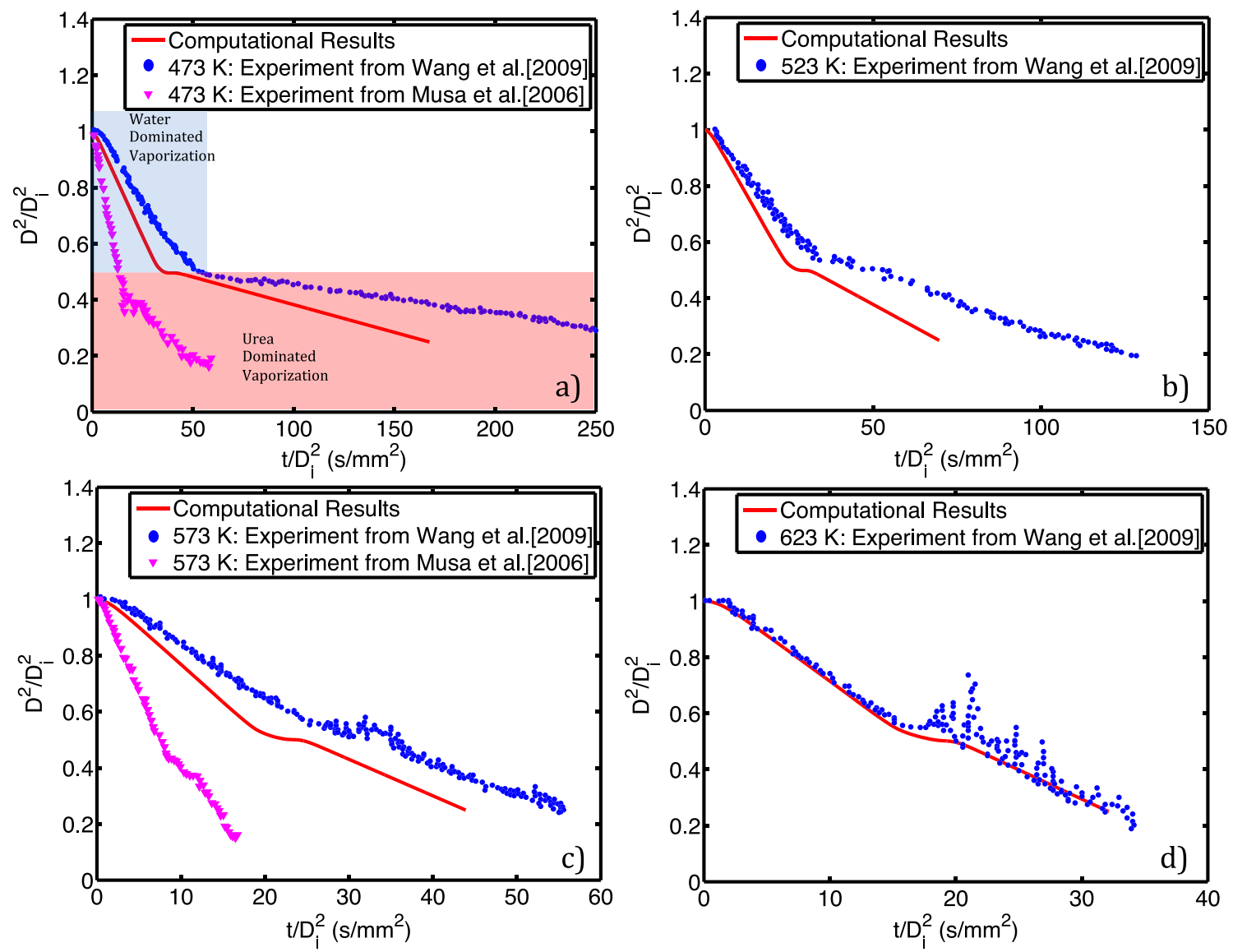

Fig. 7 UWS droplet vaporization at a $473 \mathrm{~K}$, b $523 \mathrm{~K}$, c $573 \mathrm{~K}$, and d $623 \mathrm{~K}$

between the initial period dominated by $\mathrm{C}_{7} \mathrm{H}_{16}$ vaporization, and the latter period controlled by $\mathrm{C}_{10} \mathrm{H}_{22}$.

\section{UWS Droplet Vaporization Behavior}

Numerical predictions corresponding to UWS droplet vaporization are compared to data from Wang et al. [39] in Figs. 7 and 8. The initial composition corresponds to an eutectic mixture of $32.5 \%$ urea mass fraction in water. The temperature range extends from 473 to $823 \mathrm{~K}$ under atmospheric pressure conditions. Data from Musa et al. [27] is also added to this comparison for temperatures corresponding to 473,573 , and $673 \mathrm{~K}$. This data has been discussed in the work of Kontin et al. [23] and Ebrahimian et al. [11], and hence motivates its inclusion in the present work. Both experimental data sets $[27,39]$ are performed in an electric furnace with droplets suspended from a quartz fiber. The calculation of droplet diameter differs between the papers, however, as Wang et al. [39] uses a computer program to assign diameter to each image, and Musa et al. [27] uses the diameters taken from each image and calculates the minor and major diameters of both the droplet and the fiber head, which are used in a generalized diameter. It should be noted that no modifications have been made to the thermophysical data to manipulate the matching with measurements.

Overall, reasonable agreement is observed between the numerical predictions and data from Wang et al. [39]. This is particularly the case for higher temperatures, e.g., $T \geq 623 \mathrm{~K}$, and for the initial period dominated by water vaporization. As highlighted in Fig. 7a, the vaporization process takes place beginning with a water-controlled phase and followed by a urea-governed period. The demarcation between these two periods is manifested by a change in slope of the $\mathrm{D}^{2}$ curve, similar to the previous case of a $\mathrm{C}_{7} \mathrm{H}_{16} / \mathrm{C}_{10} \mathrm{H}_{22}$ droplet. The transition region between these two slopes occurs due to the formation of a highly concentrated liquid shell of urea around the remaining water within the droplet, which is observed in experiments [31, 39].

The noticeable difference between the measurements produced by Wang et al. [39] and Musa et al. [27] do raise some questions regarding the validity of the data. In this respect, it should be noted that the paper by Chauveau et al. [8] raises the same type of questions in the vaporization of hydrocarbon fuel droplets, as explained previously. The issue is again the width of the fiber used to suspend the 

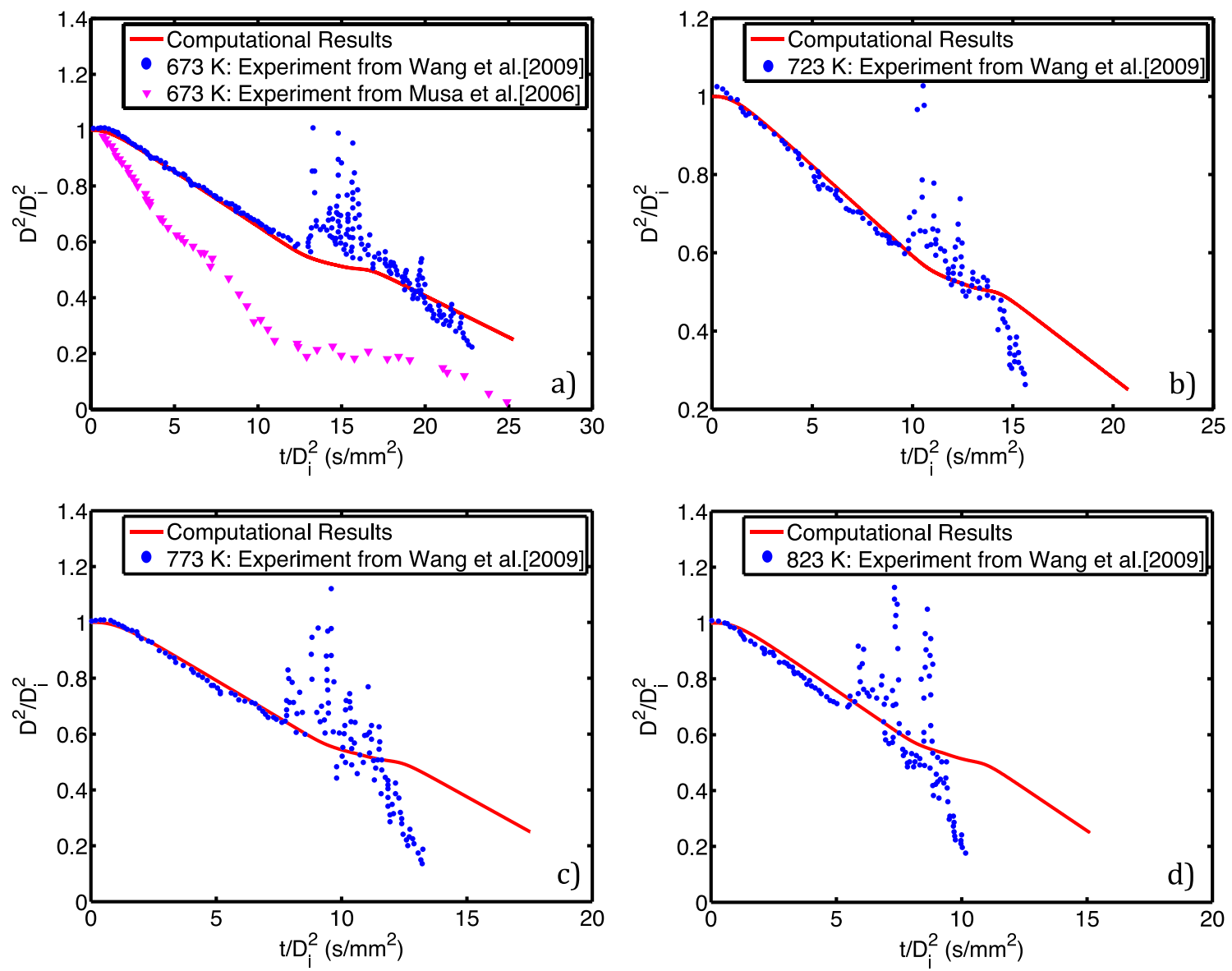

Fig. 8 UWS droplet vaporization at a 673 K, b 723 K, c 773 K, and d 823 K

droplets during the measurements and the type of arrangement used. In the experiments of Wang et al. [39], the ratio of suspension wire to droplet diameter is, $D_{\text {wire }} / D_{\text {drop }}=$ [ 0.11 to .165], while for Musa et al. [27], this parameter is not reported. Regardless, due to consistent reference in the computational literature $[3,11,25]$ to the experiments of Wang et al. [39], we interpret these measurements as being closer to the actual values, and consequently employ them as benchmark cases for the numerical comparisons.

At temperatures beginning with the $623 \mathrm{~K}$ case and higher, the erratic behavior of the droplet clearly becomes apparent in the urea-dominated phase. This is exhibited by large variations in the $D^{2} / D_{i}^{2}$ curves. Photographs of the droplet during this phase Wang et al. [39] make obvious the large distortions from sphericity observed at this stage, along with the presence of micro-explosions violently disturbing the gas-liquid interface. In view of these phenomena, the assumptions leading to the governing equations presented in Section 2 no longer hold and the comparison with the data is no longer meaningful. In fact, at this stage, it is irrelevant to consider a unique value for $D^{2} / D_{i}^{2}$ to use as a metric. Hence, results from numerical computations are no longer reported during this late period of the droplet lifetime.

Kontin et al. [23], Musa et al. [27], and Wang et al. [39] all discuss internal boiling or micro-explosions of these droplets. Wang et al. [39] states that the micro-explosions may be due to low species diffusion between water and urea inside the droplet. Motivated by a need to better understand the conditions leading to micro-explosion, the next section considers in more detail the internal evolution of the temperature and species fields within the droplet.

\subsection{Droplet Interior Distribution of Thermodynamic States}

A common topic in various studies in the literature [3, $23,27,39]$ is the manifestation of micro-explosions in UWS droplets. The experiments reported by Musa et al. [27] showed that boiling, expansion, and crystallization are present during the transition between the water and urea-dominated vaporization phase. Wang et al. [39] documents large bubbles forming and collapsing during the vaporization of the droplets, and state that this is caused by water exceeding its boiling point within the droplet. 
They also note that this process may be aggravated by diffusional resistance between the water and urea. Kontin et al. [23] reports urea solidification on the surface of the droplet in their experiment. In each of these experiments, a solid urea shell is reported forming on the surface, following by the superheating of water and the resulting nucleation and micro-explosion. Abu-Ramadan et al. [3] studied this phenomena, testing the viability using Raoult's law to measure effective boiling temperature of the water at the center. They concluded that the nucleation rate was too low when compared to the diffusional transportation rate to cause micro-explosions. However, the overwhelming experimental evidence of micro-explosion from the results of Musa et al. [27] and Wang et al. [39] is in contradiction of this conclusion.

To numerically study the dynamics within the droplet leading to an environment where micro-explosions are likely, we consider the interior of a droplet as consisting of a number of thermodynamic states undergoing change. In essence, each computational cell along with its temperature, pressure, and chemical composition can be considered to represent a local thermodynamic state. Furthermore, the evolution of each state can be traced within the context of the urea-water phase diagram. This phase diagram, shown in Fig. 9 [12], consists of three key regions, namely the water solidification, the urea-water liquid solution, and the urea solidification region. In the next set of plots, results corresponding to the evolution of the thermodynamic state of the droplet interior (most inner computational cell) and droplet interface (liquid cell bordering the gas-liquid interface) are plotted corresponding to temperatures $473,623,723$, and $823 \mathrm{~K}$, at atmospheric pressure. This is shown in Fig. 11. Time is indicated within these figures and is presented normalized by $\tau_{C I S}$. Here, $\tau_{C I S}$ corresponds to the instant when a change in the temperature slope with respect to time occurs and helps in

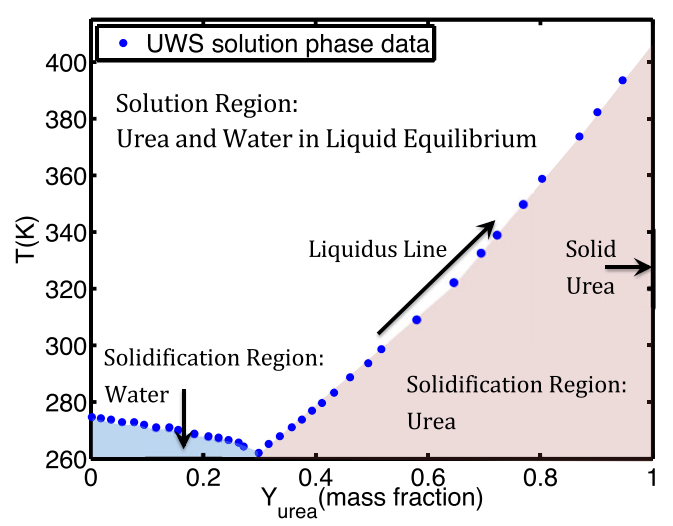

Fig. 9 Binary phase diagram at atmospheric pressure for urea-water solution [12] identifying the underlying transition between the water and urea-dominated vaporization, as demonstrated in Fig. 10. The trajectories within the phase diagram can be described as occurring in three distinct phases. In the first phase, the entire droplet interior undergoes a rise in temperature at almost constant composition, i.e., it remains at nearly the initial eutectic state. This takes place over a brief period of time ranging from $0.125 \tau_{C I S}$ to $0.5 \tau_{C I S}$ depending on temperature. The second phase occurs at a nearly constant temperature and represents a migration of the entire droplet towards the urea-solidification region. At lower temperatures, entrance into this region occurs even before the transition into the urea-dominated vaporization period has begun, i.e., $t<\tau_{C I S}$. Moreover, at lower temperatures, the entire droplet enters the urea-solidification region, while at higher temperatures, the droplet interior remains within the liquid solution region and only the surface has urea solidification. It should be noted that within this region, the solid is $100 \%$ urea and the liquid has a composition consistent with the liquidus line at the same temperature.

The final stage begins at $t \approx \tau_{C I S}$ and is characterized by a departure from the quasi-steady temperature towards higher values, as well by a more gradual enrichment of urea. If the interior of the droplet has entered the ureasolidification region, during this last stage, it will typically exit this domain back into the liquid solution region, albeit at much higher temperature. Compared to Fig. 9, a similar plot is shown by Ebrahimian et al. [11], where they compared their numerical results to the numerical results of Kontin et al. [23] taken at $673 \mathrm{~K}$ and at 1 bar in a nitrogen environment. However, Ebrahimian et al. [11] employed a rapid mixing model, and hence could not capture with this model changes in urea enrichment occurring at various spatial locations within the droplet.

To examine the urea-solidification process in more detail, Fig. 12 shows results in terms of species mass fraction vs

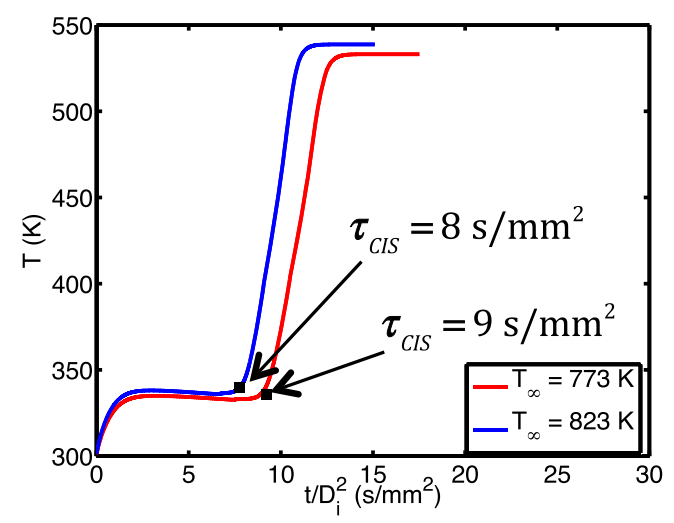

Fig. 10 Surface temperature over droplet lifetime, for $T_{\infty}=$ $773 K$ and $T_{\infty}=823 \mathrm{~K}$ 
Fig. 11 Evolution of droplet interior and free surface thermodynamic states
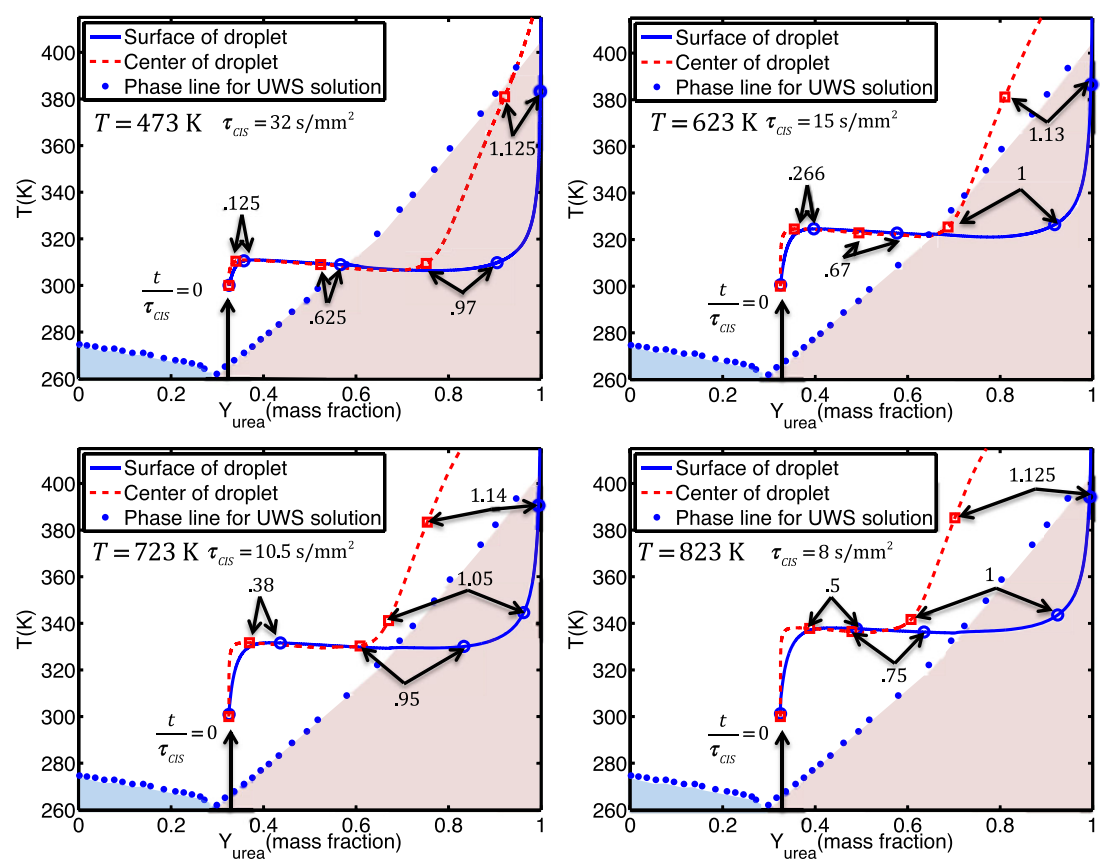

dimensionless radius as a function of time. The blue lines indicate that the local thermodynamic state falls within the liquid solution region, while a red " $X$ " indicates that the state is within the urea solidification region. The first apparent trend in this plot is the enrichment of urea throughout the entire droplet with time. A second observation is that with increasing ambient temperature, a smaller fraction of the droplet enters the solidification region. This becomes obvious when considering the shrinkage of the red " $\mathrm{X}$ " area at higher temperatures. Beyond a threshold temperature ranging between 623 and $723 \mathrm{~K}$, the central core of the droplet only sees a solution region, i.e., it never solidifies.

The entrapment of liquid in the central region of the droplet, particularly at higher temperature is evident from this figure, is accompanied by a large increase in temperature (see Fig. 11). The consequence is an associated increase in pressure, since the external surface of the droplet has solidified and hence the pressure cannot easily equilibrate. To estimate the rise in pressure associated with this phenomena, we compute the change in pressure considering
Fig. 12 Evolution of the distribution of thermodynamic state of the droplet at various ambient temperatures
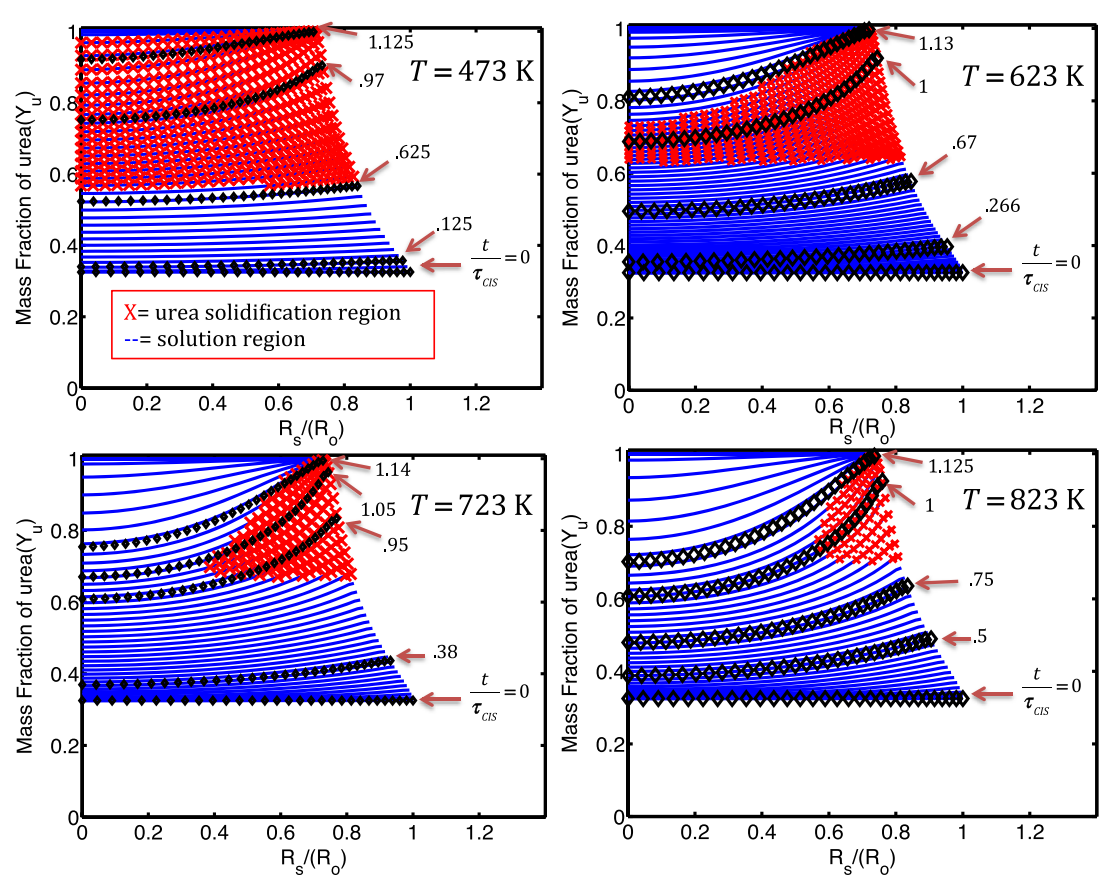
Fig. 13 Comparison of $D^{2}$ slope vs time $(623 \mathrm{~K})$ with varying $\operatorname{Re}$

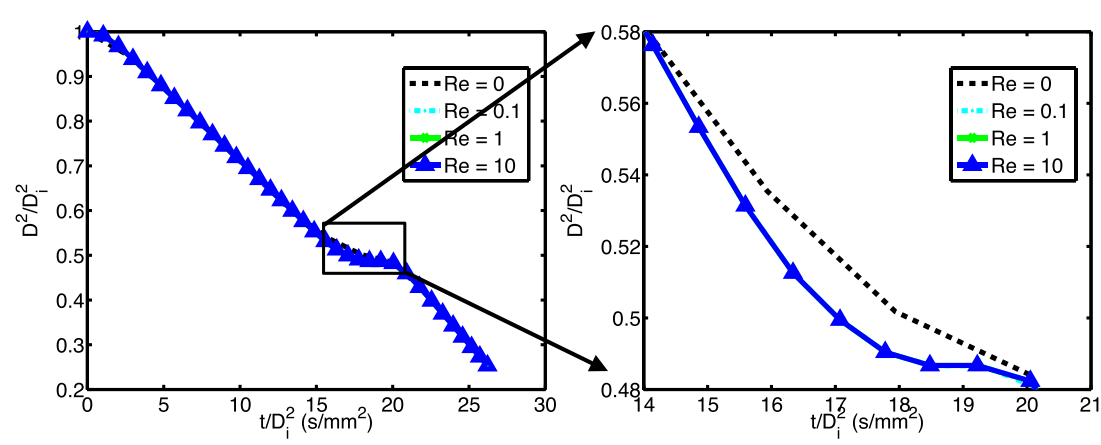

that the density remains fixed (assuming the external urea shell cannot expand) and that the temperature rises as indicated in Fig. 11. The calculations employ the PR-EOS and the estimated pressure difference is $\approx 10,000 \mathrm{~Pa}$. This can be interpreted as the likely culprit in the generation of micro-explosions.

If the rapid mixing model considered in the literature $[2,3,11,25]$ did apply, the thermodynamic state trajectories between the surface and center of the droplet would coincide perfectly. This would render the case of liquid entrapment within the droplet an unattainable situation and would remove the possibility of internal pressure rise. Consequently, the driving force leading to the micro-explosion would not be present. As shown in Figs. 11 and 12, the rapid mixing state does not occur and significant differences between urea mass fraction within the droplet are established.

An alternative view of the non-uniformity of the urea mass fraction profiles, given the rise to the entrapment and subsequent potential micro-explosion, is examined by considering the time scales involved. An estimate of the vaporization time scale of vaporization can be formed by considering the jump condition for energy in the quasisteady vaporization state (11d). In this state, the heat flux originating from the liquid side and the liquid velocity at the interface can be considered to be negligible $\left(D^{2}\right.$ law conditions), and hence the time scale for vaporization becomes

$\tau_{\text {vap }}=\frac{2 \rho^{l} R_{s}^{2} L_{v a p, m i x}}{N u k_{a i r}\left(T_{\infty}-T_{s}\right)}$.

The species diffusion time scale can be approximated by $\tau_{\text {diff }}=\left(2 R_{s}\right)^{2} / D_{a b}$. The ratio between these times scales $\tau_{\text {vap }} / \tau_{\text {diff }}$ ranges from approximately 0.9 to 0.3 corresponding to $T_{\infty}=473$ and $823 \mathrm{~K}$, respectively. Hence, the vaporization time scale is always smaller than the diffusion time scale and becomes even smaller at higher ambient temperatures. This implies that with increasing temperature, the presence of a sharper urea concentration gradient becomes more pronounced, which eventually leads to urea solidification at the interface. Results corroborating this assertion can be found in Figs. 11 and 12.

\subsection{Mixing}

The effect of hydrodynamic mixing on the droplet vaporization characteristics is accounted in the present work by incorporating the models advanced by Sirignano [33] and Abramzon and Sirignano [1] for both heat and mass diffusion (through $\mathscr{D}_{a b, e f f}$ and $k_{\text {eff }}^{l}$ ). To gauge the impact that the mixing produced by aerodynamics effects has on the droplet vaporization behavior, various values of the (liquid) Reynolds numbers, $R e^{l}=0.1,1$, and 10 are considered. The results are shown in Fig. 13 revealing that liquid internal mixing has a minor role in the vaporization characteristics. This is due to the low species diffusion value, $\mathscr{D}_{a b, e f f}$, when compared to the thermal diffusivity, $\alpha_{e f f}^{l}$, yielding an effective Lewis number that is always greater than 10 for lower $R e^{l}$ values and reaches a value approximately equal to 30 for $R e^{l}=10$. With this high value for Lewis number, the dynamics for mass diffusion are considerably slower than those from heat diffusion, even at this relatively large value of $R e^{l}$. The consequence is again a sharp gradient in urea mass fraction, urea solidification, and the conditions conducive of micro-explosion. It should be noted that this study was only for internal mixing, which influences both the species mass fraction and the internal energy governing equations.

\section{Conclusion}

After systematically verifying and validating the computational solver used in droplet vaporization calculation, it has been employed in the study of the physics governing urea-water-solution droplet vaporization. The focus of this work provides fundamental knowledge towards the improvement or development of more macroscopic computational models involving urea-water-solution sprays and their dynamics within a UREA-SCR after-treatment configuration. The main findings from this work are 
summarized as follows along with suggestions for future research:

1. Reasonable agreement is achieved with the $D^{2}$ data from Wang et al. [39], with the model being able to capture relatively well the water-dominated and subsequent urea-dominated vaporization period.

2. By considering the evolution of thermodynamic states within the droplet, it is possible to characterize UWS droplet vaporization as occurring in three distinct phases: (1) an overall rise in temperature at essentially constant composition, (2) an overall enrichment in urea at almost constant temperature, and (3) departure from a quasi-steady temperature of the second phase and gradual enrichment of urea, particularly near the interface, leading to solidification conditions.

3. The ambient temperature plays a particularly strong role in shaping the local mass fraction and temperature state particularly during the third phase of vaporization. At higher temperatures, internal species gradients are slow to equilibrate, resulting in large urea mass fraction gradients and solidification of urea at the interface. Thus, the entrapment of urea becomes more noticeable at these higher temperatures.

4. $D^{2}$ time histories have historically been used to assess vaporization models. As noted in the literature [8], the arrangement used in suspending the droplets during experimental measurements can add a considerable amount of uncertainty in the results due to conductive heat transfer process taking place between the suspension wires and the droplet. This has important implications in UWS droplet vaporization due to the fact that in addition to vaporization, a solidification process is also taking place, which is highly sensitive to heat transfer. More experimental data sets for UWS droplet vaporization paying careful attention to this aspect would benefit future model validation efforts.

5. Even though the rapid mixing model for droplet vaporization, used for instance in $[2,3,25]$, may produce $D^{2}$ time histories that agree reasonably well with experimental data, it is unable to generate the gradients in urea mass fraction that lead to urea solidification at the interface. This solidification is reported here and has been also reported in the experimental works of Musa et al. [27], Wang et al. [39], and Kontin et al. [23]. We believe along with others [39] that it is a necessary condition for the production of micro-explosions.

6. The effect of aerodynamic forcing caused by the movement of UWS droplets has been studied within the context of effective diffusivity models [1, 33]. It is reported that internal mixing has a negligible effect on the $D^{2}$ time history curves, even at relatively high values for $R e^{l}$.

Acknowledgments We are grateful to support from the U.S. Department of Energy, award no. DE-EE0000202.

\section{Appendix A: Peng-Robinson Equation of State}

Coefficients A and B are given by

$A=\frac{a_{m} P}{\left(R_{\text {univ }} T\right)^{2}} \quad$ and $\quad B=\frac{b_{m} P}{R_{\text {univ }} T}$.

The mixing rules, $a_{m}$ and $b_{m}$, are defined using pure component parameters, and at most one binary interaction parameter,

$a_{m}=\sum_{j}^{N_{s p}} \sum_{k}^{N_{s p}} X_{j} X_{k} \sqrt{a_{j} a_{k}}\left(1-k_{j k} P\right) \quad$ and $\quad b_{m}=\sum_{j=1}^{N_{s p}} X_{j} b_{j}$.

The binary interaction coefficient, $k_{j k}$, is,

$k_{j k}=1-\left[\frac{2\left(v_{c r i t, j} v_{c r i t, j}\right)^{1 / 6}}{\left(v_{c r i t, j}\right)^{1 / 3}\left(v_{c r i t, k}\right)^{1 / 3}}\right]$,

and the pure component parameters $a_{j}$ and $b_{j}$ are defined using the critical temperature and pressure of species $\mathrm{j}$. They are

$$
a_{j}=0.45724 \frac{\left(R_{\text {univ }} T_{\text {crit }, j}\right)^{2}}{P_{\text {crit }, j}} \alpha_{P R, j}
$$

and $\quad b_{j}=0.07780 \frac{R_{u n i v} T_{c r i t, j}}{P_{\text {crit }, j}}$.

Table 2 Parameters corresponding to the Peng-Robinson Equation of State solution

\begin{tabular}{lllllll}
\hline Critical properties & nHeptane & Decane & Water & Urea & Air \\
$T_{\text {crit }}(K)$ & 540.26 & 617.70 & 647.13 & 892 & $\begin{array}{l}\text { Nitrogen } \\
126.1\end{array}$ \\
\hline$P_{\text {crit }}($ bar $)$ & 27.36 & 21.10 & 220.55 & 90.50 & 37.71 & 33.94 \\
$v_{\text {crit }}\left(\frac{\mathrm{cm}^{3}}{\mathrm{~mol}}\right)$ & 431.9 & 624 & 56 & 218 & 92.35 & 90.1 \\
$M\left(\frac{\mathrm{g}}{\mathrm{mol}}\right)$ & 100.204 & 142.285 & 18.06 & 60.06 & 28.96 & 28.013 \\
$\omega(-)$ & 0.351 & 0.492 & 0.345 & 0.620 & 0.040 & 0.040 \\
\hline
\end{tabular}


The parameter $\alpha_{P R, j}$ in turn is equal to unity at the critical temperature and is a function of $\omega$, reduced temperature, and $m_{j}$, given as

$$
\sqrt{\alpha_{P R, j}}=1+m_{j}\left(1-\sqrt{T_{r, j}}\right) .
$$

Here, $m_{j}$ is an empirical function of the acentric factor and $T_{r}$ is the reduced temperature, respectively, given by

$$
\begin{aligned}
m_{j} & =0.37464+1.54226 \omega_{j}+0.26992 \omega_{j}^{2} \\
\text { and } \quad T_{r} & =\frac{T}{T_{c r i t, j}} .
\end{aligned}
$$

The critical properties and the acentric factor for urea, water, air, nitrogen, $\mathrm{C}_{7} \mathrm{H}_{16}$, and decane, barring the critical temperature of urea, were taken from Yaws [40].

\section{Appendix B: Thermophysical Equations}

Latent heat of vaporization, thermal conductivities, and specific heats are provided in the following set of equations. For the latent heat of vaporization, we employ:

$$
\begin{aligned}
L_{\text {vap }, \text { urea }} & =\left(A_{L u}\left(1-T / B_{L u}\right)^{n_{L u}}\right), \\
L_{\text {vap }, H_{2} O} O & =\left(A_{L w}\left(1-T / B_{L w}\right)^{n_{L w}}\right), \\
L_{\text {vap }, C_{7} H_{16}} & =\left(A_{L h}\left(1-T / B_{L h}\right)^{n_{L h}}\right), \\
L_{\text {vap }, \text { dec }} & =\left(A_{L h}\left(1-T / B_{L h}\right)^{n_{L h}}\right) .
\end{aligned}
$$

For the liquid thermal conductivities:

$$
\begin{aligned}
k_{l, C_{7} H_{16}=} & \left(\left(\left(\left(f_{k l h} T+e_{k l h}\right) T\right.\right.\right. \\
& \left.\left.\left.\left.+d_{k l h}\right) T+c_{k l h}\right) T+b_{k l h}\right) T+a_{k l h}\right), \\
k_{l, \text { decane }}= & A_{k l d}+B_{k l d} T+C_{k l d} T^{2}, \\
k_{l, \text { urea }}= & A_{k l u}+B_{k l u} T+C_{k l u} T^{2}, \\
k_{l, H_{2} O} O & \left(\left(\left(\left(f_{k l w} T+e_{k l w}\right) T\right.\right.\right. \\
& \left.\left.\left.\left.+d_{k l w}\right) T+c_{k l w}\right) T+b_{k l w}\right) T+a_{k l w}\right) .
\end{aligned}
$$

For the gas thermal conductivities:

$$
\begin{aligned}
k_{g, \text { nitrogen }} & =\left(A_{k n}+B_{k n} T_{g}+C_{k n} T_{g}^{2}\right), \\
k_{g, \text { air }} & =\left(A_{k a}+B_{k a} T_{g}+C_{k a} T_{g}^{2}\right), \\
k_{g, \text { decane }} & =\left(A_{k g d}+B_{k g d} T_{g}+C_{k g d} \cdot * T_{g} \cdot{ }^{2}+D_{k g d} T_{g}{ }^{3}\right), \\
k_{g, C_{7} H_{16}} & =\left(A_{k g h}+B_{k g h} T_{g}+C_{k g h} T_{g} \cdot{ }^{2}+D_{k g h} T_{g} \cdot{ }^{3}\right), \\
k_{g, \text { urea }} & =\left(A_{k g u}+B_{k g u} T_{g}+C_{k g u} T_{g}^{2}+D_{k g u} T_{g}^{3}\right), \\
k_{g, H_{2} O}= & \left(A_{k g w}+B_{k g w} T_{g}+C_{k g w} T_{g}^{2}+D_{k g w} T_{g}^{3}\right) .
\end{aligned}
$$

Lastly, for the specific heats:

$$
\begin{aligned}
c_{p, C_{7} H_{16}} & =a_{c h}+b_{c h} T+c_{c h} T^{2}+d_{c h} T^{3}, \\
c_{p, \text { decane }} & =A_{c d}+B_{c d} T+C_{c d} T^{2}+D_{c d} T^{3}+E_{c d} T^{4}, \\
c_{p, \text { urea }} & =A_{c u}+B_{c u} T+C_{c u} T^{2}+D_{c u} T^{3}, \\
c_{p, H_{2} O} O & =\left(\left(\left(\left(f_{c w} T+e_{c w}\right) T+d_{c w}\right) T+c_{c w}\right) T+b_{c w}\right) T+a_{c w} .
\end{aligned}
$$

where the constants for all of these equations can be found in Yaws [40].

\section{B.1 Thermophysical Properties}

The diffusion coefficients for both liquid and gas phase were calculated using the general hard sphere diffusion assumption found in Poling et al. [30]. For the liquid, we have,

$\mathscr{D}_{a b}^{l, o}=\frac{7.4 \bullet 10^{-8}\left(\Phi M_{b}\right)^{1 / 2} T}{\eta_{b} V_{a}^{0.6}}$,

where $\eta_{b}$ is the viscosity of solvent $b, V_{a}$ is the molar volume of solute $A$ at its normal boiling temperature, and $\Phi$ is the association factor of solvent $b$ which is 2.6 for water, as noted in Poling et al. [30]. To calculate the diffusion coefficient for a binary mixture, the following equation is used

$\mathscr{D}_{a b}^{l}=\left[\left(\mathscr{D}_{a b}^{l, o}\right)^{x_{b}}\left(\mathscr{D}_{b a}^{l, o}\right)^{x_{a}}\right] \alpha$,

where $\alpha$ is a correction factor used for liquids. Note that all cases considered have at most two chemical species in the liquid. Air is only considered in the phase equations, and its mass fraction in liquid is essentially zero and therefore not calculated within the droplet.

The gas diffusion equation, as shown in Poling et al. [30], Fuller and Giddings [17], Fuller et al. [18], and Fuller et al. [16] is

$$
\mathscr{D}_{\text {ab }}^{g}=14.3 e-4 \bullet T_{1 / 3 l a w}^{1.75} \cdot \frac{\sqrt{1 / M_{\mathrm{H}_{2} O \text { or urea }}+1 / M_{\text {air }}}}{p_{\text {atm }}\left(\Sigma_{H_{2} O \text { or urea }}^{1 / 3}+\Sigma_{\text {air }}^{1 / 3}\right)^{2}} .
$$

The critical temperature for urea was calculated, based on data from Emel'yanenko et al. [14], as well as Bernhard et al. [5], using

$$
T_{\text {crit }}=T_{\text {boil }}\left[0.584+0.965 \sum_{k} N_{k}(t c k)-\left(\sum_{k} N_{k}(t c k)\right)^{2}\right]^{-1} \text {, }
$$

which is the Joback method, discussed in Poling et al. [30].

The governing equations generally pertain to mixtures of different chemical species. In this context, the following variables need to be prescribed for the mixture and are calculated as follows:

$$
\begin{gathered}
L_{v a p, \text { mix }}=\frac{\sum_{i}^{N_{s p}} L_{v a p, i} X_{i}^{v}}{\sum_{i}^{N_{s p}} X_{i}^{v}}, \\
k_{\text {mix }}^{l}=Y_{a} k_{a}+Y_{b} k_{b}-\alpha_{k_{\text {mix }}^{l}}\left(k_{b}-k_{a}\right)\left[1-\left(k_{b}\right)^{1 / 2}\right] k_{b},
\end{gathered}
$$


$k_{\text {air }, \text { mix }}=\frac{\left(X_{\mathrm{H}_{2} O}^{g} k_{\mathrm{H}_{2} \mathrm{O}}^{g}+X_{\text {urea }}^{g} k_{\text {urea }}^{g}+X_{\text {air }}^{g} k_{\text {air }}^{g}\right)}{\left(X_{\mathrm{H}_{2} \mathrm{O}}^{g}\left(A_{u, w}+A_{a, w}\right)+X_{\text {urea }}^{g}\left(A_{w, u}+A_{a, u}\right)+X_{\text {air }}^{g}\left(A_{w, a}+A_{u, a}\right)\right)}$,

and

$C_{p, \text { mix }}=\sum_{k} Y_{k} C_{p, k}, \quad$ for $\mathrm{k}=1: N_{s p}$.

\section{References}

1. Abramzon, B., Sirignano, W.: Droplet vaporization model for spray combustion calculations. Int. J. Heat. Mass Transfer. 32, 1605-1618 (1989)

2. Abu-Ramadan, E., Saha, K., Li, X.: Modeling of the injection and decomposition processes of urea-water-solution spray in automotive scr systems. SAE International (2011)

3. Abu-Ramadan, E., Saha, K., Li, X.: Modeling the depleting mechanism of urea-water-solution droplet for automotive selective catalytic reduction systems. AIChE 57(11), 3210-3225 (2011)

4. ACEA: Acea report on selective catalytic reduction: the most promising technology to comply with imminent euro iv and euro $\mathrm{v}$ emissions standards for hd engines. Technical report (2003)

5. Bernhard, A.M., Czekaj, I., Elsener, M., Wokaun, A., Krocher, O.: Evaporation of urea at atmospheric pressure. J. Phys. Chem. A 115, 2581-2589 (2011)

6. Byron Bird, R., Stewart, W.E., Lightfoot, E.N.: Transport Phenomena. 2nd. John Wiley (2002)

7. Birkhold, F., Meingast, U., Wassermann, P., Deutschmann, O.: Modeling and simulation of the injection of urea-water-solution for automotive scr denox-systems. Appl. Catal. B Environ. 70, 119-127 (2007)

8. Chauveau, C., Halter, F., Lalonde, A., Gokalp, I.: An experimental study on the droplet vaporization: effects of heat conduction through the support fiber. ILASS-EUROPE (2008)

9. Coulson, J.M., Richardson, J.F., Backhurst, J.R., Harker, J.H. Coulson and Richardson's Chemical Engineering Volume 1 Fluid Flow,Heat Transfer and Mass Transfer, 6th. Elsevier (1999)

10. Daif, A., Bouaziz, M., Chesneau, X., Ali Cherif, A.: Comparison of multicomponent fuel droplet vaporization experiments in forced convection with the sirignano model. Exp. Thermal Fluid Sci. 18, 282-290 (1999)

11. Ebrahimian, V., Nicolle, A., Habchi, C.: Detailed modeling of the evaporation and thermal decomposition of urea-water solution in scr systems. AIChE (2011)

12. Egal, M.: Structure and properties of cellulose / NAOH aqueous solutions, gels and regenerated objectS. $\mathrm{PhD}$ thesis, ecole des mines de PARIS (2006)

13. Elliot, J.R., Lira, C.T.: Introductory Chemical Engineering Thermodynamics.Prentice Hall (2012)

14. Emel'yanenko, V.N., Kabo, G.J., Verevkin, S.P.: Measurement and prediction of thermochemical properties: improved increments for the estimation of enthalpies of sublimation and standard enthalpies of formation of alkyl derivatives ofurea. J. Chem. Eng. Data 51, 79-87 (2006)

15. Fang, H.L., Dacosta, H.F.M.: Urea thermolysis and nox reduction with and without scr catalysts. Appl. Catal. B: Environmen. 46, 17-34 (2003)
16. Fuller, E.N., Ensley, K., Giddings, J.C.: Diffusion of halogenated hydrocarbons in helium. The effect of structure on collision cross sections. J. Phys. Chem. 73 (1969)

17. Fuller, E.N., Giddings, J.C.: A comparison of methods for predicting gaseous diffusion coefficients. J. Gas Chromatogr. 3(222) (1965)

18. Fuller, E.N., Schettler, P.D., Giddings, J.C.: New method for prediction of binary gas-phase diffusion coefficients. Ind. Eng. Chem. 58(5), 18 (1966)

19. Givler, S., Abraham, J.: Supercritical droplet vaporization and combustion studies. Prog. Energy Combst. Sci. 22, 1-28 (1996)

20. Johnson, T.V.: Diesel emissions in review. SAE Int. J. Engines 4(1), 143-157 (2011)

21. Kim, J.Y., Ryu, S.H., Ha, J.S.: Numerical prediction on the characteristics of spray-induced mixing and thermal decomposition of urea solution in scr system. Fall Technical Conference of the ASME Internal Combustion Engine Division, ICEF2004-889 (2004)

22. Klein, S., Nellis, G.: Thermodynamics. Cambridge University Press (2012)

23. Kontin, S., Hofler, A., Koch, R., Bauer, H.J.: Heat and mass transfer accompanied by crystallization of single particles containing urea-water-solution. ILASS Europe (2010)

24. Kucera, A., Hill, J.: On inward solidifying cylinders and spheres initially not at their fusion temperature. Int. J. Non-Linear Mech. 1, 73-82 (1986)

25. Lundstrom, A., Waldheim, B., Strom, H., Westerberg, B.: Modelling of urea gas phase thermolysis and theoretical details on urea evaporation. Engineers, Part D: Journal of Automobile Proceedings of the Institution of Mechanical Engineering (2011)

26. Munnannur, A., Liu, Z.G.: Development and validation of a predictive model for def injection and urea decomposition in mobile scr denox systems. SAE International (2010)

27. Musa, S., Saito, M., Furuhata, T., Arai, M.: Evaporation characteristics of a single aqueous urea solution droplet. ICLASS 195 (2006)

28. Nomura, H., Ujhe, Y., Rath, H.J., Sato, J., Kono, M.: Experimental study on high-pressure droplet evaporation using microgravity conditions. Twenty-Sixth Symposium on Combustion, pp. 12671273 (1996)

29. Peng, D., Robinson, D.: A new two-constant equation of state. Ind. Eng. Chem. Fundam 15(1), 59-64 (1976)

30. Poling, B.E., Prausnitz, J.M., O'Connell, J.P.: The Properties of Gases and Liquids, Fifth Edition. McGraw-Hill, New York (2001)

31. Koch, R., Kontin, S., Bauer, H.J.: Size and refractive index measurements of inhomogeneous particles with a solid crust by phase doppler anemometry. ILASS-EUROPE (2011)

32. Schaber, P.M., Colson, J., Higgins, S., Thielen, D., Anspach, B., Brauer, J.: Thermal decomposition (pyrolysis) of urea in an open reaction vessel. Thermochim. Acta 424, 131-142 (2004)

33. Sirignano, W.A.: Fluid dynamics and transport of droplets and sprays. Cambridge University Press, Cambridge (1999)

34. Smyth, E.: Molecular orientation and the partial vapor pressures of binary mixtures, i. systems composed of normal liquids. J. Am. Chem. Soc. 51(9) (1929) 
35. Ström, H., Lundström, A., Andersson, B.: Choice of urea-spray models in cfd simulations of urea-scr systems. Chem. Eng. J. 150, 69-82 (2009)

36. Torres, D.J., Trujillo, M.F.: Kiva-4: An unstructured ale code for compressible gas flow with sprays. J. Compuational Phys. 219, 943-975 (2006)

37. Trujillo, M.F., Torres, D.J., O’Rourke, P.: High-pressure multicomponent liquid sprays : departure from ideal behaviour. Int. J. Engine Res. 5, 229-246 (2004)
38. Twigg, M.V.: Progress and future challenges in controlling automotive exhaust gas emissions. Appl. Catal. B Environ. 70, 2-15 (2007)

39. Wang, T.J., Baek, S.W., Lee, S.Y.: Experimental investigation on evaporation of urea-water-solution droplet for scr applications. AIChE 46, 17-34 (2009)

40. Yaws, C.L.: Chemical Properties Handbook. Knovel (1999)

41. Zhu, G., Aggarwal, S.: Transient supercritical droplet evaporation with emphasis on the effects of equation-of-state. Int. J. Heat Mass Transfer. 43, 1157-1171 (2000) 